\title{
EDITORIAL
}

\section{Immune checkpoint inhibitors and irAEs}

\author{
Fukumi Furukawa ${ }^{1,2}$ \\ ${ }^{1}$ Takatsuki Red Cross Hospital, Takatsuki City, Osaka, Japan \\ ${ }^{2}$ Wakayama Medical University, Wakayama City, Wakayama, Japan
}

Keywords: immune checkpoint inhibitor; PD-1; CTLA-4; immune-related adverse events

ARTICLE INFO

Received: June 25, 2018

Available online: June 30, 2018

*CORRESPONDING AUTHOR

Fukumi Furukawa, Takatsuki Red

Cross Hospital, Osaka, Japan;

ffurukawa@takatsuki.jrc.or.jp

\section{CITATION}

Furukawa F. Immune checkpoint inhibitors and irAEs. Trends Immunother 2018; 2(1): 930. doi: 10.24294/ti.v2.i2.930

\section{COPYRIGHT}

Copyright (C) 2018 by author(s) and EnPress Publisher LLC. This work is licensed under the Creative Commons Attribution-NonCommercial 4.0 International License (CC BY-NC 4.0). http://creativecommons.org/licenses/ by $/ 4.0 /$
In the development of medicine, there are not many cases that advanced in a direction different from the original purpose. Sometimes it is practical in areas that no one expected. The immune checkpoints programmed cell death 1 (PD-1) is exactly that example. Dr. Hiroyuki Nishimura showed the development of lupus-like autoimmune diseases by disruption of the $P D-1$ gene encoding an ITIM motif-carrying immunoreceptor ${ }^{[1]}$. Because of the wide range of ligand distribution in the body, its biological significance pervades almost every aspect of immune responses including autoimmunity, tumor immunity, infectious immunity, transplantation immunity, allergy and immunological privilege ${ }^{[2]}$. Immune checkpoints inhibitors (ICIs) have opened promising avenues in the treatment of cancer. Various blocking antibodies targeting PD-1 and cytotoxic T-lymphocyte-associated protein 4 (CTLA-4) are approved for human use. They significantly improved disease outcome in a number of cancer patients by boosting anti-tumor immune responses. As Seidel et al. described in their review article ${ }^{[3]}$, mortality among advanced stage patients and the frequency of treatment-related adverse events remain high with current treatment.

ICIs were developed and put to practical use in the unexpected course, although science is sometimes like that. Furthermore, it is also noteworthy that unexpected side effects appear, even when it becomes better to be administered to many cancer patients. In this issue, Inaba et al. reported immune-related adverse effects (irAEs) including endocrine dysfunctions have been reported ${ }^{[4]}$. It is well known that dysfunctions in the pituitary gland and the thyroid gland by ICIs are often observed, and those in the adrenal glands and the pancreas are less frequent. Unfortunately, the mechanisms of endocrine irAEs by ICIs, however, remain unclear, and optimal prevention, prediction, and treatment of the irAEs are still uncertain.

In addition, the need for predictive markers of treatment efficacy and the development of improved treatment avenues therefore remain as acute as ever $^{[3]}$. sCD163 and CXCL5 may serve as possible prognostic biomarkers for irAEs in patients with advanced melanoma treated with nivolumab ${ }^{[5]}$.

Taken together, we believe that these subjects will be soon resolved because a number of world-wide studies are underway for these subjects in many organs.

\section{Acknowledgments}

This work was supported by a grant from the Japan Society for Promotion of Science. 


\section{Conflicts of interest}

No conflict of interest was reported by the author.

\section{References}

1. Nishimura H, Nose M, Hiai H, et al. Development of lupus-like autoimmune diseases by disruption of the $P D-1$ gene encoding an ITIM motif-carrying immunoreceptor. Immunity 1999; 11(2): 141-151. doi: 10.1016/S1074-7613(00)80089-8.

2. Okazaki T, Honjo T. PD-1 and PD-1 ligands: From discovery to clinical application. Int Immunol 2007; 19(7): 813-824. doi: 10.1093/intimm/dxm057.
3. Seidel JA, Otsuka A, Kabashima K. Treating tumors with immune checkpoint inhibitors: Rationale and limitations. Trends Immunother 2017; 1(1): 2-9. doi: 10.24294/ti.v1.i1.20.

4. Inaba $\mathrm{H}$, Ariyasu $\mathrm{H}$, Okuhira $\mathrm{H}$, et al. Endocrine dysfunctions during treatment of immune-checkpoint inhibitors. Trends Immunother 2018; 2(2). doi: 10.24294/ti.v2.i2.606.

5. Fujimura T, Sato Y, Tanita K, et al. Serum levels of soluble CD163 and CXCL5 may be predictive markers for immune-related adverse events in patients with advanced melanoma treated with nivolumab: a pilot study. Oncotarget 2018; 9(21): 15542-15551. doi: 10.18632/oncotarget.24509. 